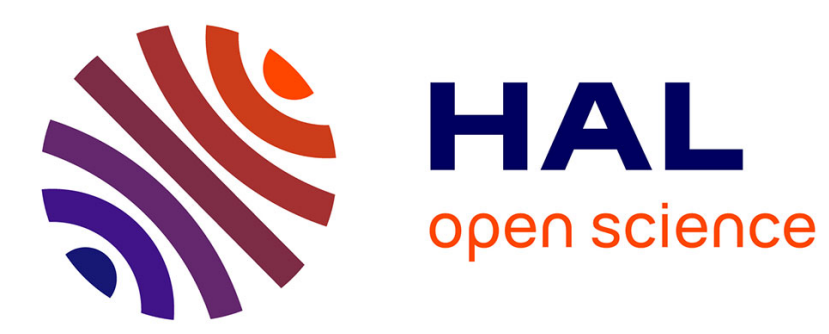

\title{
On the interpolation of electromagnetic near field without prior knowledge of the radiating source
}

Benjamin Fuchs, L.L. Coq, M.D. Migliore

\section{To cite this version:}

Benjamin Fuchs, L.L. Coq, M.D. Migliore. On the interpolation of electromagnetic near field without prior knowledge of the radiating source. IEEE Transactions on Antennas and Propagation, 2017, 65 (7), pp.3568-3574. 10.1109/TAP.2017.2705163 . hal-01622625

\section{HAL Id: hal-01622625 \\ https://hal-univ-rennes1.archives-ouvertes.fr/hal-01622625}

Submitted on 21 Jan 2021

HAL is a multi-disciplinary open access archive for the deposit and dissemination of scientific research documents, whether they are published or not. The documents may come from teaching and research institutions in France or abroad, or from public or private research centers.
L'archive ouverte pluridisciplinaire HAL, est destinée au dépôt et à la diffusion de documents scientifiques de niveau recherche, publiés ou non, émanant des établissements d'enseignement et de recherche français ou étrangers, des laboratoires publics ou privés. 


\title{
On the Interpolation of Electromagnetic Near Field without Prior Knowledge of the Radiating Source
}

\author{
Benjamin Fuchs, Senior Member, IEEE Laurent Le Coq and Marco Donald Migliore, Senior Member, IEEE
}

\begin{abstract}
A method is proposed to interpolate the electromagnetic near field when no information on the radiating source is available. In absence of a priori knowledge, general properties of the electromagnetic field are exploited to estimate the field, namely the minimum complexity of the field and the continuity of the first derivatives. These properties are enforced by minimizing the nuclear norm and using the Thin Plate Spline interpolation results, respectively. The proposed procedure is validated experimentally by interpolating the planar electrical near field radiated by three antennas. The quality of the interpolation and its robustness to noise is investigated. Despite its simplicity, the interpolation method is able to properly estimate the near field from a random coarse sampling of $2 \lambda$. The quality of the near field interpolation is also confirmed by deriving the far field. These promising results pave the way for the development of fast antenna measurement procedures.
\end{abstract}

Keywords: Antenna measurements, sampling methods, inverse problems.

\section{INTRODUCTION}

Interpolation of electromagnetic field on general manifolds is a relevant and challenging problem both theoretically and practically. Most field interpolation or reconstruction approaches rely on the proper exploitation of some a-priori information on the electromagnetic source. Broadly speaking, the stronger the a-priori information on the source is, the easier the interpolation is. Several powerful techniques exploiting various a priori on the antennas have been successfully applied to significantly reduce the number of observed data for field interpolation and reconstruction purposes. The approach followed in [1] introduces the shape, position and dimension of the source as a-priori information in order to obtain a number of samples that depends on the source geometry. The number of samples is approximately equal to $\Sigma /(\lambda / 2)^{2}$, wherein $\Sigma$ is the area of the minimum convex surface surrounding the source, and $\lambda$ is the free space wavelength. A sampling method for array diagnostic was proposed in [2]-[4]. The approach, based on compressed sensing/sparse recovery techniques, allows to reduce significantly of the number of measurements, that is then not a function of the source but only of the number of failures of the array. In [5], numerical simulations of the Antenna Under Test are used to obtain a proper basis for the radiated field, allowing a large reduction of the samples.

However, in many realistic and practical scenarios, no reliable information on the radiating sources are available. This

Manuscript received xx, 2016; revised xx, xx.

B. Fuchs and L. Le Coq are with the IETR / University of Rennes I, France. (e-mail: \{benjamin.fuchs;laurent.le-coq\}@univ-rennes1.fr). M.D. Migliore is with the DIEI, University of Cassino e Lazio Meridionale, Italy. (e-mail mdmiglio@unicas.it). is the case when measuring electromagnetic radiation whose source characteristics cannot be known or seen, e.g. because of a radome or when the antenna is mounted on a complex structure contributing to the radiation.

This paper is a first attempt to investigate the interpolation of the electromagnetic near field radiated by completely unknown sources. This interpolation is not exact since the amount of available information is not sufficient to perfectly reconstruct the field, and the problem at hand is rather the estimation of the field distribution from incomplete and noisy data.

The solution of this 'interpolation' problem without a priori knowledge uses the weighted minimization of two norms that embody some general properties of any electromagnetic field. We first minimize the nuclear norm of the matrix collecting the values of the field on a uniform observation grid. Physically, it amounts to select the field having 'minimum complexity' which is a reasonable assumption in presence of uncertainty on the data and no knowledge on the electromagnetic source. The measure of the linear complexity of the field is given by the rank of the matrix whose entries are the values of this field on the observation surface, as detailed in [6].

The second term is the $\ell_{2}$-norm of the difference between the field on the rectangular grid and an estimation of the field obtained by the Thin Plate Spline (TPS) interpolation algorithm [7], [8]. Despite its simplicity and efficiency, the TPS technique is the one providing the best field interpolation results among the interpolation schemes investigated by the authors. It is a generalization of the 1-dimensional cubic splines to the two-dimensional space. The values obtained by the TPS interpolation are considered affected by uncertainty, therefore they are weighted in the minimization procedure according to their accuracy.

The proposed interpolation algorithm is applied to experimental data obtained from planar near-field measurements of various antennas using a reduced set of randomly selected data. It is useful to stress out that near-field measurements are used as a test case to show the performance of the interpolation technique. In principle, this approach can be applied in any case when the interpolation of electromagnetic field data without any knowledge on the source is required.

The paper is organized as follows. The interpolation algorithm is described in Section II. The physical meaning of the proposed regularizers and their limitations are discussed. In Section III, the proposed interpolation algorithm is applied on near field planar measurements and experimentally assessed. Three antennas with various features are considered: a standard gain horn working at $60 \mathrm{GHz}$, a Gaussian beam horn working at $60 \mathrm{GHz}$ and a reflector antenna working at $10 \mathrm{GHz}$. The in- 
terpolated near fields are compared to the exact data and the far field patterns are also derived. Conclusions and perspectives are drawn in Section IV.

\section{INTERPOLATION TECHNIQUE}

The proposed interpolation algorithm combines two regularizers while the measured field is to be respected up to an accuracy that depends on the noise level. These two regularizers are first described. Their physical meaning, advantages and drawbacks are discussed before introducing the complete interpolation algorithm.

\section{A. Interpolation by Nuclear Norm Minimization}

Let $\mathbf{X} \in \mathbb{C}^{m \times n}$ be the unknown matrix collecting the electromagnetic field radiated by a source on a given uniform lattice $\Omega$ of dimension $m$ by $n$. This field is measured at $p$ positions of $\Omega(p<m . n)$ and collected in the vector $\mathbf{e} \in \mathbb{C}^{p}$ In order to estimate the field on each point of $\Omega$ we use the following rank minimization algorithm:

$$
\min _{\mathbf{X} \in \mathbb{C}^{m \times n}} \operatorname{rank}(\mathbf{X}) \text { subject to }\|\mathcal{A}(\mathbf{X})-\mathbf{e}\|_{2} \leq \varepsilon
$$

where $\mathcal{A}$ is the linear map: $\mathbb{C}^{m \times n} \rightarrow \mathbb{C}^{p}$ that selects the $p$ measured points among the m.n sampling points of $\Omega$, and where the parameter $\varepsilon$ depends on the noise level affecting the measured data $\mathbf{e}$.

The problem (1) is known as matrix completion problem [9]. In general, Eq. (1) is a challenging non-convex optimization problem for which all known finite time algorithms have at least doubly exponential running times in both theory and practice. A recent heuristic introduced in [10] minimizes the nuclear norm, or the sum of the singular values of the matrix, over the constraint set. The nuclear norm of a matrix $\mathbf{X}$ of rank $r$ reads:

$$
\|\mathbf{X}\|_{*}=\sum_{k=1}^{r} \sigma_{k}(\mathbf{X})
$$

where $\sigma_{k}(\mathbf{X})$ denotes the $k$-th largest singular value of $\mathbf{X}$. This heuristic is a good convex surrogate of the rank function as detailed in [10], [11]. Whereas the rank function counts the number of non-zero singular values, the nuclear norm sums their amplitude. In some sense, the nuclear norm is to the rank functional what the convex $\ell_{1}$-norm is to the $\ell_{0}$-norm in the sparse recovery area. This interesting analogy between the matrix rank and nuclear norm minimization problems and the vector sparsity and $\ell_{1}$ norm problems is detailed in [12].

The problem (1) can thus be approximated using the heuristic optimization:

$$
\min _{\mathbf{X} \in \mathbb{C}^{m \times n}}\|\mathbf{X}\|_{*} \text { subject to }\|\mathcal{A}(\mathbf{X})-\mathbf{e}\|_{2} \leq \varepsilon
$$

For a general matrix $\mathbf{X} \in \mathbb{C}^{m \times n}$, which may not be positive definite or even symmetric, the nuclear norm heuristic can be expressed in a positive semidefinite form [10] and the problem
(3) is then equivalent to:

$$
\begin{aligned}
& \min _{\mathbf{X}, \mathbf{Y}, \mathbf{Z}} \operatorname{Tr}(\mathbf{Y})+\operatorname{Tr}(\mathbf{Z}) \\
& \text { subject to }\left\{\begin{array}{l}
\|\mathcal{A}(\mathbf{X})-\mathbf{e}\|_{2} \leq \varepsilon \\
{\left[\begin{array}{cc}
\mathbf{Y} & \mathbf{X} \\
\mathbf{X}^{*} & \mathbf{Z}
\end{array}\right] \succeq 0}
\end{array}\right.
\end{aligned}
$$

where $\mathbf{Y}$ and $\mathbf{Z}$ are Hermitian matrices of dimension $m \times m$ and $n \times n$ respectively. Any semidefinite programming solver can be used to find the best solutions of (4). We use the software CVX [13] that calls an interior point optimizer. The lines of code to solve (4) are given in Appendix A.

Solving (4) amounts to find the minimum rank matrix $\mathbf{X}$, i.e. the simplest representation, compatible with the available data. The physical meaning of this choice is related to the 'complexity' of the electromagnetic source radiating the field. In order to clarify this point, let us consider the radiation operator $\mathcal{F}$ (compact under quite general hypothesis) relating the currents on the unknown source to the field on the observation domain [14]-[16]. The effective rank of $\mathcal{F}$ is called the Number of Degrees of the Field (NDF), it corresponds to the effective number of dimensions of the range of $\mathcal{F}$. The NDF depends on the electrical dimension of the source, its shape and the observation domain, as detailed in [17]. Broadly speaking, the higher the $N D F$ is, the more 'complex' the field radiated by the source is, wherein the complexity is measured by the number of basis required to represent the field, as explained in [6].

As an illustrative example, let us consider a $2 a \times 2 a$ square planar current source having constant amplitude and phase that is placed at a distance $d$ from a $2 L \times 2 L$ observation domain, see Fig. 1(c). The variation of the NDF versus $a / \lambda$ is plotted as red circles versus $d$ in Fig. 1(a). The values are normalized in order to range from 0 to 1 when $a / \lambda$ varies from 1 to 5 . As expected, the NDF increases with the electrical dimension of the source. Let us consider the radiated field $E(x, y)$ on the observation domain. We plot the variation of the normalized nuclear norm (a good approximation of the rank) of $E(x, y)$, blue curve of Fig. 1(a). The normalized NDF and nuclear norm of $E(x, y)$ behave similarly. This trend is confirmed when investigating the influence of the distance $d$ between the source and the observation domain as shown in Fig. 1(b).

Accordingly, in absence of a-priori information on the source (such as its electrical dimension), the interpolation found by the nuclear norm minimization (4) is associated to the 'simplest' electromagnetic source.

\section{B. Thin Plate Spline Interpolation}

The Thin Plate Spline (TPS) belongs to the class of polyharmonic splines and is the mathematical version of the physical spline used the last century: a thin wooden strip hold in place at a number of points that represent 'known values'. The strip assumes the shape that minimizes the total strain energy, giving an interpolation curve belonging to the $C^{1}$ continuity class, i.e. continuous with its first partial derivatives. The TPS has the same physical interpretation. Let us consider a thin metallic plate that is slightly bent so that its deflection $f(\mathbf{r})$, $\mathbf{r}=(x, y)$ is constraint to assume height $z_{i}$ in a discrete set 


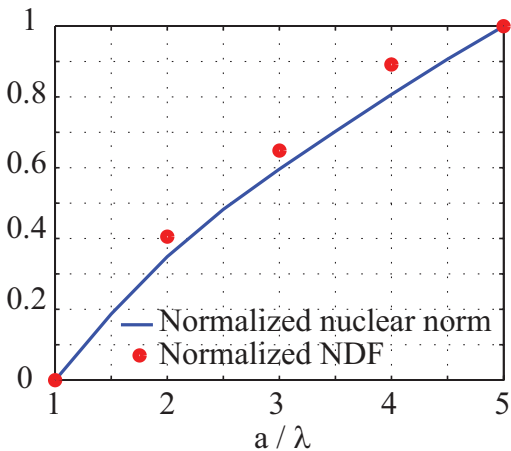

(a)

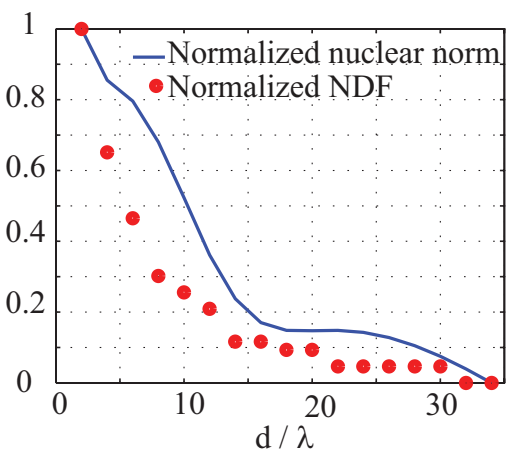

(b)

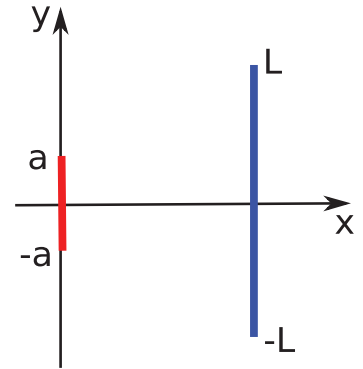

(c)

Fig. 1. Variation of the NDF (red points) and of the nuclear norm (blue curve) versus (a) the length $2 a$ of the side of a square source; $d=5 \lambda ; L=20 \lambda$ and (b) the distance $d$ between the source and the observation domain; $2 a=2 \lambda ; L=20 \lambda$. The plotted quantities are normalized to range between 0 and 1. (c) Representation of the geometry of the problem with the associated notations.

of points $\mathbf{r}_{i}=\left(x_{i}, y_{i}\right)$, i.e. $f\left(\mathbf{r}_{i}\right)=z_{i}, i=1, \ldots, n$. The form of the metallic plate will minimize the bending energy [18]:

$$
\iint_{\mathbb{R}^{2}}\left(\frac{\partial^{2} f}{\partial x^{2}}+2 \frac{\partial^{2} f}{\partial x \partial y}+\frac{\partial^{2} f}{\partial y^{2}}\right) d x d y
$$

From a numerical point of view, the interpolation function $f(\mathbf{r})$ is obtained efficiently by solving a linear system. Let us represent the thin plate interpolation $f(\mathbf{r})$ as a superposition of radial basis functions $\Phi(r)$ weighted by some coefficients $c_{i}$ :

$$
f(\mathbf{r})=\sum_{i=1}^{p} c_{i} \Phi\left(\left|\mathbf{r}-\mathbf{r}_{i}\right|\right) .
$$

The radial basis functions $\Phi(r)=-r^{2} \log \left(r^{2}\right), r=$ $\sqrt{x^{2}+y^{2}}$, are the Green's functions of the biharmonic operator $\nabla^{4}$. The coefficients $c_{i}$ are obtained solving the square linear system:

$$
e_{k}=\sum_{i=1}^{p} c_{i} \Phi\left(\left|\mathbf{r}_{k}-\mathbf{r}_{i}\right|\right), \quad k=1, \ldots, p
$$

where $e_{k}$ are the known values of the field at the positions $\mathbf{r}_{k}$. The interpolated values of the field at the positions $\mathbf{r}_{i}$ are $\tilde{e}_{i}=f\left(\mathbf{r}_{i}\right), i=1, . ., m . n-p$.

\section{Interpolation Algorithm}

The proposed algorithm combines the two previously described interpolation techniques.

The nuclear norm minimization (4) tends to underestimate the value of the field when an important sequence of adjacent data are missing. Consequently, the interpolation by nuclear norm minimization behaves well until a 'phase transition' value after which the probability of having a sequence of missed values becomes important, inducing a fast increase of the interpolation error. To overcome this drawback, we use the field interpolated via the TPS using (6) and (7) and denoted $\tilde{\mathbf{e}}$. We consider the values of $\tilde{\mathbf{e}}$ that are significantly higher than the noise level more reliable and use accordingly weights proportional to the amplitude of $\tilde{\mathbf{e}}$. The proposed interpolation algorithm is then:

$$
\begin{aligned}
& \min _{\mathbf{X} \in \mathbb{C}^{m \times n}}\|\mathbf{X}\|_{*}+\alpha\left\|\mathbf{W}\left(\mathcal{A}^{c}(\mathbf{X})-\tilde{\mathbf{e}}\right)\right\|_{2} \\
& \text { subject to }\|\mathcal{A}(\mathbf{X})-\mathbf{e}\|_{2} \leq \varepsilon
\end{aligned}
$$

The linear map $\mathcal{A}^{c}: \mathbb{C}^{m \times n} \rightarrow \mathbb{C}^{m . n-p}$ selects the $m . n-p$ points that are not measured on $\Omega$. The diagonal matrix $\mathbf{W}$ contains the weights $\left(\mathbf{W}_{i, i}=\left|\tilde{e}_{i}\right|^{-1}\right)$ and the scalar $\alpha>0$ is the regularization parameter. Its choice, a well known issue in regularization problems, is discussed in Section III-C. It can be made by tracing out the trade-off curve varying $\alpha$ over $(0,+\infty)$

It is important to note that the algorithm treats in a different way the data measured on the uniform lattice, that are associated to an explicit constraint fixed by the measurement noise level, and the data interpolated via TPS.

\section{EXPERIMENTAL RESUlts}

The goal is to interpolate from only $p$ measurements made at random positions on a uniform grid $\Omega$, the electromagnetic field at the m.n points of $\Omega$ where $m . n>p$. To measure the decimation of the field, we compute the sampling step between measurement points defined as the average cartesian distance between measurement points. In order to guarantee the randomness of the field measurement positions, all presented results are averaged values over 10 simulations.

The exact and interpolated electromagnetic fields are the complex matrices $\mathbf{X}$ and $\widetilde{\mathbf{X}}$ of dimension $m \times n$, respectively. The quality of the interpolation is assessed by computing the relative error between the exact and interpolated fields as follows:

$$
\varepsilon(\mathbf{X}, \widetilde{\mathbf{X}})=\frac{\|\mathbf{X}-\tilde{\mathbf{X}}\|_{F}}{\|\mathbf{X}\|_{F}}
$$

where $\|\cdot\|_{F}$ stands for the Frobenius norm.

Moreover and despite considering experimental data already corrupted by measurement noise, the robustness of the interpolation with respect to noise is evaluated by adding a white Gaussian noise to the measured data: $\mathbf{e}_{n}=\mathbf{e}+\mathbf{n}$. The level of the noise is quantified by the Signal-to-Noise Ratio (SNR) that is defined from the maximum received field 


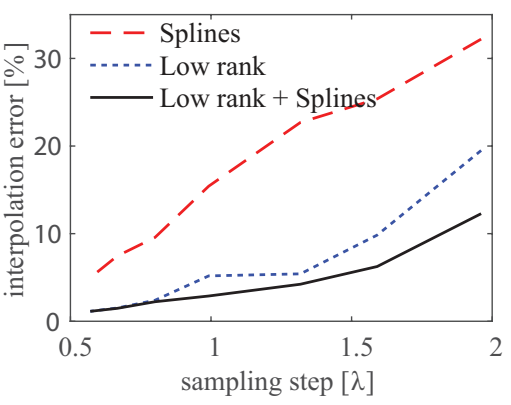

(a)

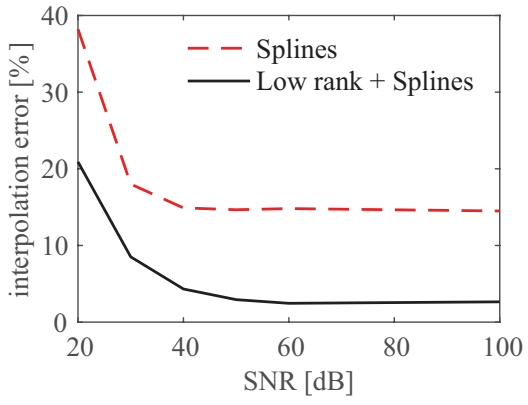

(b)

Fig. 2. Interpolation results of the near field radiated by a standard gain horn at $60 \mathrm{GHz}$. Relative interpolation error as a function of (a) the average sampling step (in absence of noise) and (b) the SNR (for an average sampling step of $1 \lambda$ ) for the spline interpolation and the proposed approach.
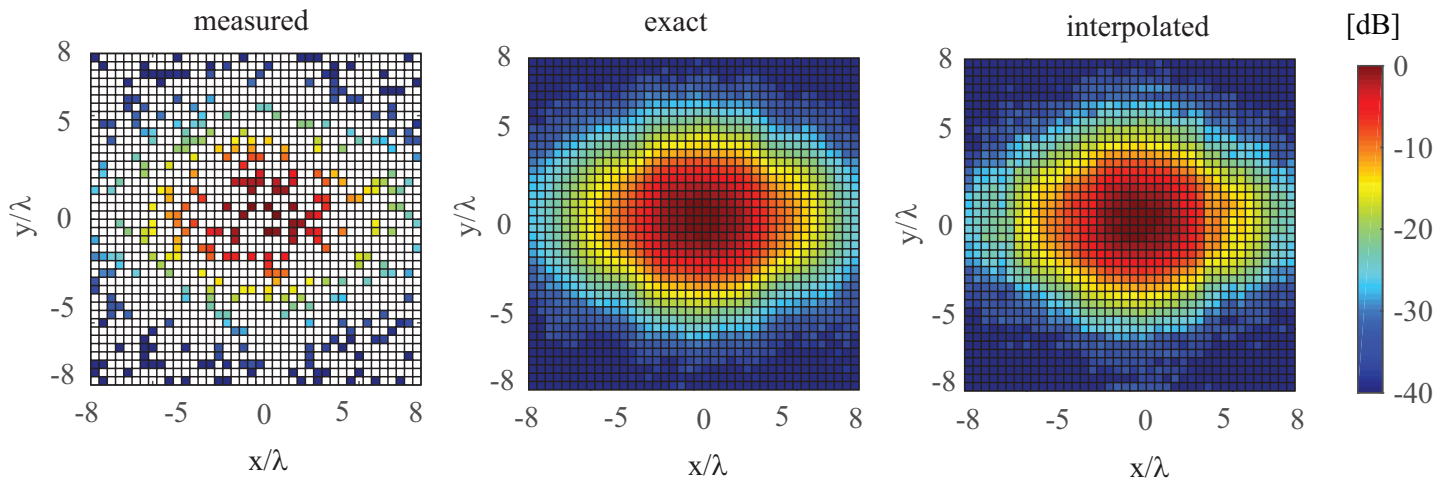

Fig. 3. Measured electric near field amplitude of a standard gain horn at $60 \mathrm{GHz}$ : (a) randomly selected measured points corresponding to an average sampling step of $2 \lambda$ (the data not considered are in blank), (b) exact near field and (c) reconstructed one by the proposed interpolation approach.
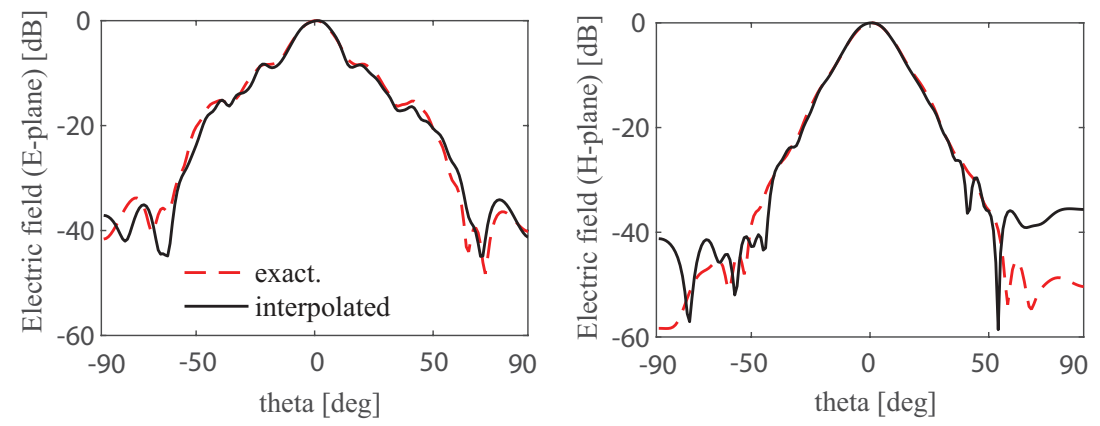

Fig. 4. (a,b) Far field patterns of the standard gain horn in both E- and H- planes computed from the exact near field (dashed line) and the interpolated one (solid line) in the case of a sampling step of $2 \lambda$.

magnitude in order to fit with the dynamic range measurement: $\mathrm{SNR}_{d B}=10 \log _{10}\left(P_{\text {signal }} / P_{\text {noise }}\right)$, where $P_{\text {signal }}$ is the maximum measured power. The bound $\varepsilon$ of the data fitting constraint in (8), is chosen to be equal to $1.1\|\mathbf{n}\|_{2}$ in all simulations. The interpolation algorithm (8) is now applied on the measured data of three antennas.

\section{A. Standard Gain Horn in V-Band}

We consider a Standard Gain Horn in V-band and measure its electrical near field radiated at $60 \mathrm{GHz}$. The sampling points are taken on a plane at a height of $5 \lambda$ from the horn aperture on a square grid of side $16 \lambda$.
The interpolation errors (9) are plotted in Fig. 2(a) as a function of the sampling step when applying the TPS, the low rank (4) and the proposed interpolation approach. The proposed interpolation approach (8) is the one providing the best interpolation results. Note that the 'smooth' field distribution (visible Fig. 3(b)) makes the low rank interpolation more efficient than the TPS technique. Besides, the proposed interpolation approach is relatively robust to noise as shown Fig. 2(b).The mappings of the electric near field magnitude (measured, exact and interpolated using (8)) are plotted in Fig. 3 . Despite a very coarse random near field sampling (step of $2 \lambda$ ), the interpolation seems to work well. This impression is 


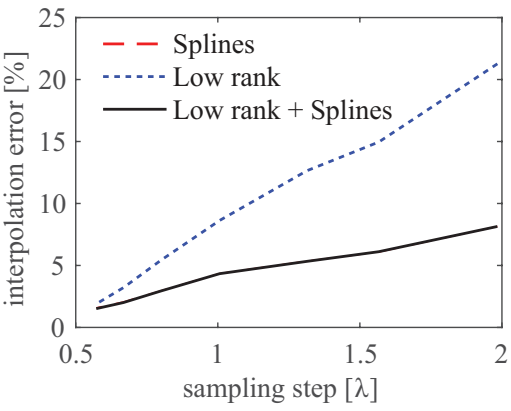

(a)

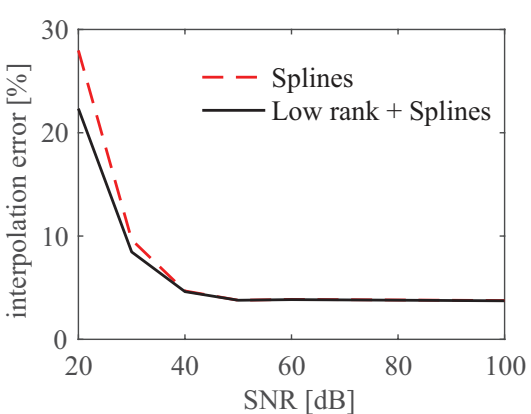

(b)

Fig. 5. Interpolation results of the near field radiated by a Gaussian beam horn at $60 \mathrm{GHz}$. Relative interpolation error as a function of (a) the average sampling step (in absence of noise) and (b) the SNR (for an average sampling step of $1 \lambda$ ) for the spline interpolation and the proposed approach.
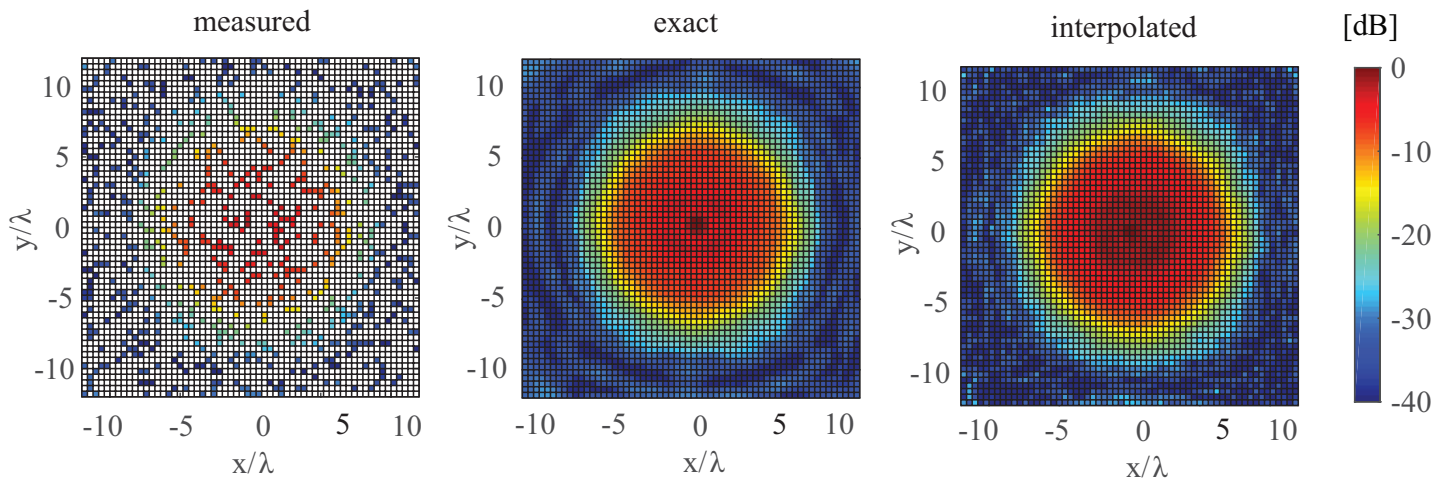

Fig. 6. Measured electric near field amplitude of a Gaussian beam horn at $60 \mathrm{GHz}$ : (a) randomly selected measured points corresponding to an average sampling step of $2 \lambda$ (the data not considered are in blank), (b) exact near field and (c) reconstructed one by the proposed interpolation approach.
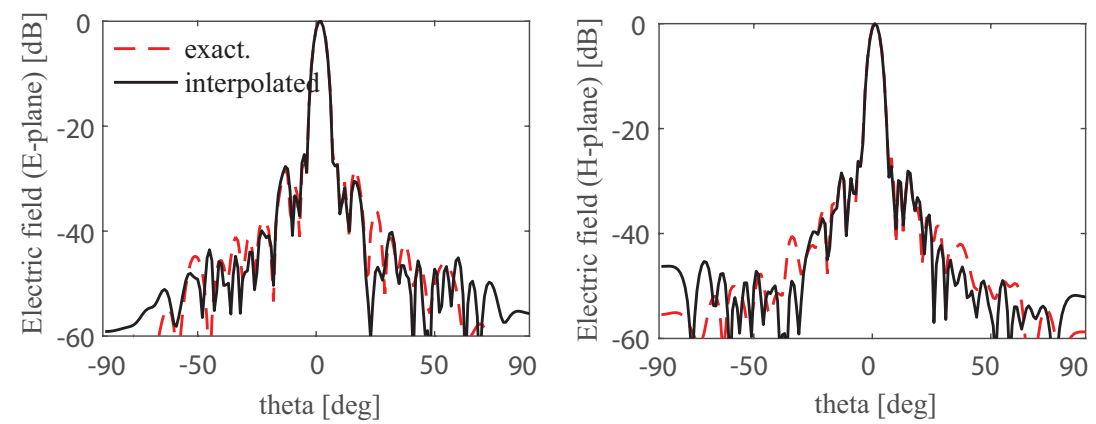

Fig. 7. (a,b) Far field patterns of a Gaussian beam horn in both E- and H- planes at $60 \mathrm{GHz}$ computed from the exact near field (dashed line) and the interpolated one (solid line) in the case of a sampling step of $2 \lambda$.

confirmed when computing the far field plotted in Fig. 4, the main beam is well approached using the interpolated results which validates the proposed approach. It means that 4 times less points than the classical half wavelength sampling can be used to properly characterize this antenna without the use of any a priori information.

\section{B. Gaussian Beam Horn in V-Band}

The same figures are used to assess the proposed interpolation scheme on a different antenna, a Gaussian beam horn whose field is sampled at $60 \mathrm{GHz}$ at a height of $5 \lambda$. Its near field is more focused than the standard gain horn as visible in Fig. 6(b). Consequently, the splines interpolation turns out to outperform the low rank interpolation as reported in Fig. 5(a) and the low rank minimization do not bring any improvement to the interpolation in this case.The proposed interpolation approach is also robust to noise as plotted in Fig. 5(b). The near field interpolation results are shown in Fig. 6 in the case of a coarse field sampling of $2 \lambda$. The far field computation results, plotted in Fig 7, confirm the quality of the interpolation despite this very coarse field sampling. 


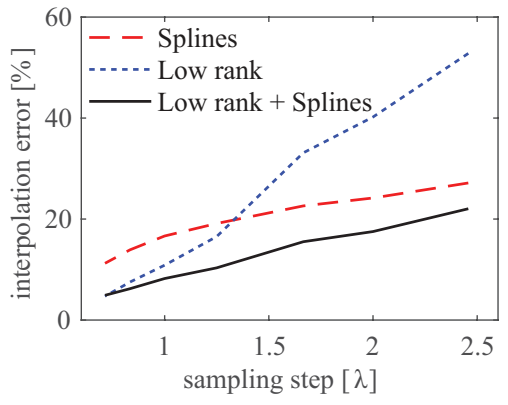

(a)

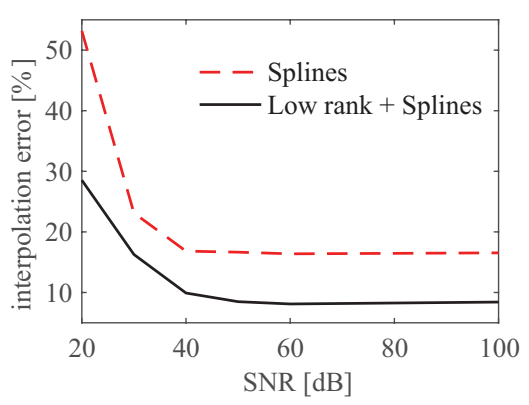

(b)

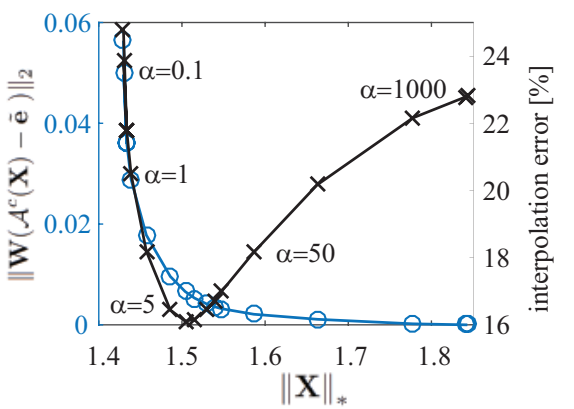

(c)

Fig. 8. Interpolation results of the near field radiated by a reflector antenna at $10 \mathrm{GHz}$. Relative interpolation error as a function of (a) the average sampling step (in absence of noise) and (b) the SNR (for an average sampling step of $1 \lambda$ ) for the spline interpolation and the proposed approach. (c) Variation of the nuclear norm as a function of the $\ell_{2}$-norm and influence of the interpolation error (trade-off $\alpha$-curve of equation (8)).
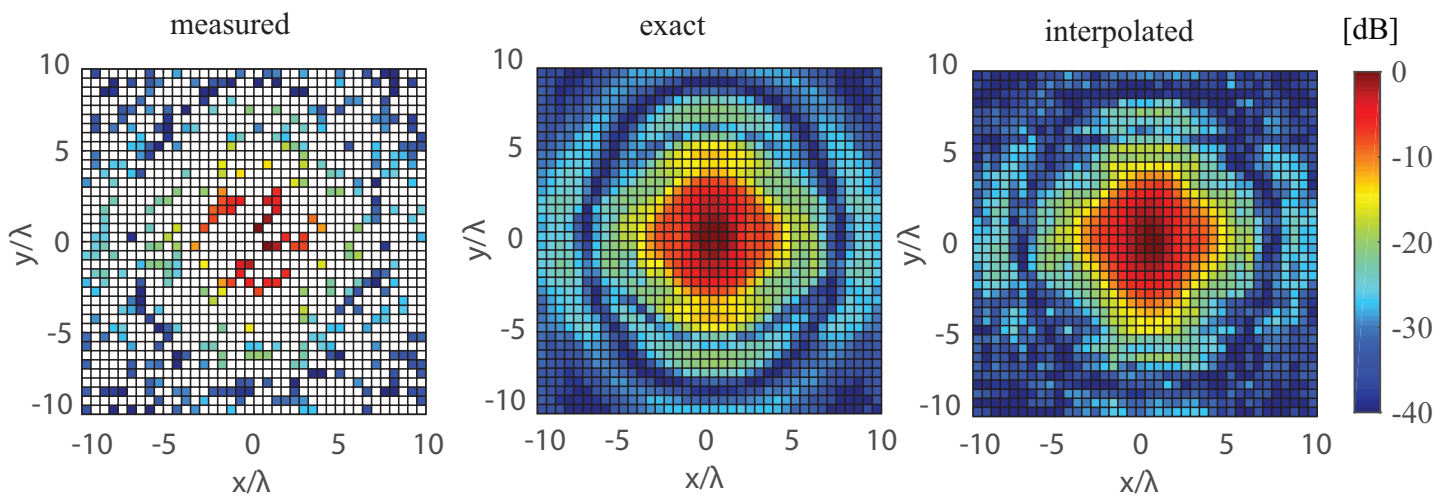

Fig. 9. Measured electric near field amplitude of a reflector antenna at $10 \mathrm{GHz}$ at a height of $7 \lambda$ : (a) randomly selected measured points corresponding to an average sampling step of $2 \lambda$ (the data not considered are in blank), (b) exact near field and (c) reconstructed one by the proposed interpolation approach.
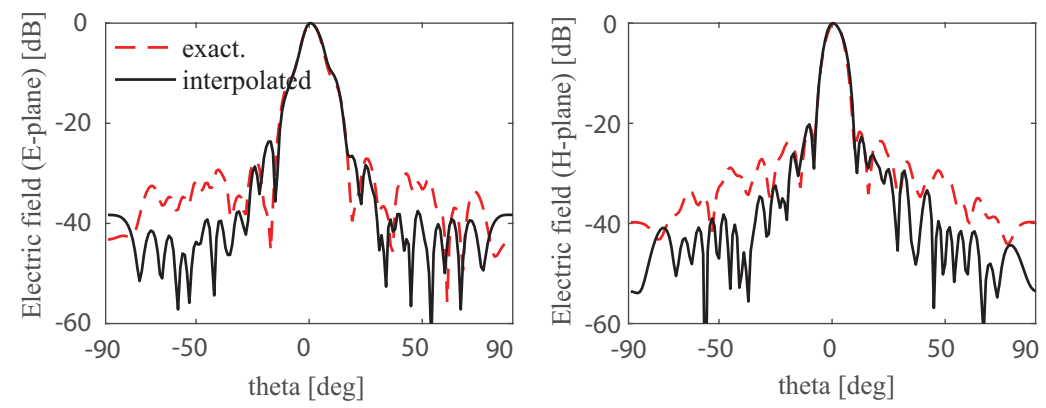

Fig. 10. (a,b) Far field patterns of a reflector antenna in both E- and $\mathrm{H}$ - planes at $10 \mathrm{GHz}$ computed from the exact near field (dashed line) and the interpolated one (solid line) in the case of a sampling step of $2 \lambda$.

\section{Reflector Antenna in X-Band}

A reflector antenna of diameter $9 \lambda$ at $10 \mathrm{GHz}$ is now considered. Its near field is sampled at a height of $7 \lambda$. The proposed approach combines the advantages of the low rank and splines interpolation as shown in Fig. 8(a) to provide a low sampling error for all sampling step despite the relatively 'complex' near field distribution (see Fig. 9(b)). The interpolation error degrades gracefully as the noise increases as reported in Fig. 8(b). The trade-off curve is plotted in Fig. 8(c). The two terms in the objective of (8) are reported as $\alpha$ varies from $10^{-2}$ to $10^{2}$. The lowest interpolation error is achieved when the curve has the maximum curvature as typically observed in similar situations. The near field mappings and far field pattern plottings from these mappings are plotted in Fig. 9 and 10 respectively. They validate the good interpolation results.

\section{CONCLUSION}

A method to interpolate the near-field distribution of antennas without a-priori information from random sampling measurements is proposed. The method is based on the weighted minimization of two terms, the residual error between the measured data and an estimation of the field obtained using 
the thin plate spline, and the nuclear norm of the field. These norms translate some general properties of any electromagnetic field, namely the continuity of its first derivatives and the minimization of its complexity, respectively. The proposed interpolation algorithm is fast, simple to implement and robust towards strong undersampling and noise as experimentally confirmed on three different kind of antennas working at $\mathrm{V}$ and $\mathrm{X}$ - bands. It is worth noting that the method has been tested from a highly-reduced random set of data to show its potential in critical cases. Note that the proposed near field is not limited to planar near field as it can be straightforwardly applied to cylindrical, spherical, polar and bi-polar sampling grid, allowing a simple and fast interpolation when the full set of data is not available and no a priori on the antenna can be exploited.

The application of the proposed approach to interpolate the electromagnetic far field is currently under investigation.

\section{APPENDIX I}

\section{A CVX CODE FOR MATRIX COMPLETION}

$\%$ Inputs

$\%(\mathrm{~m}, \mathrm{n})$ : dimensions of the unknown field matrix $\mathrm{X}$

$\%$ e: measured field at $\mathrm{p}$ random locations

$\%$ epsilon: upper bound value

$\%$ ind: vector of the $\mathrm{p}$ measurement indices among the m.n field samples

\section{cvx_begin}

variable $X(m, n)$ complex

variable $\mathrm{Y}(\mathrm{m}, \mathrm{n})$ hermitian

variable $\mathrm{Z}(\mathrm{m}, \mathrm{n})$ hermitian

$\operatorname{minimize}(\operatorname{trace}(\mathrm{Y})+\operatorname{trace}(\mathrm{Z}))$

subject to

norm( $X($ ind $)$-e ,2) <= epsilon;

$\left[\mathrm{Y}, \mathrm{X} ; \mathrm{X}^{\prime}, \mathrm{Z}\right]>=0$;

cvX_end

\section{REFERENCES}

[1] O.M. Bucci, C. Gennarelli, C. Savarese, "Representation of Electromagnetic Fields over Arbitrary Surfaces by a Finite and non Redundant Number of Samples," IEEE Trans. Antennas Propag., vol. 46, no. 3, pp.351-359, March 1998.

[2] M.D. Migliore, "On the Sampling of the Electromagnetic Field Radiated by Sparse Sources," IEEE Trans. Antennas Propag., vol. 63, no. 2, pp. 553-564, Feb. 2015.

[3] B. Fuchs, L. Le Coq, and M.D. Migliore, "Fast Antenna Array Diagnosis from a Small Number of Far-Field Measurements," IEEE Trans. Antennas Propag., vol. 64, no. 6, pp. 2227-2235, June 2016.

[4] M.D. Migliore, "A simple introduction to compressed sensing/sparse recovery with applications in antenna measurements," IEEE Antennas and Propagation Magazine, vol. 56, no. 2, pp. 14-26, April 2014.

[5] G. Giordanengo, M. Righero, F. Vipiana, G. Vecchi, M. Sabbadini, "Fast Antenna Testing With Reduced Near Field Sampling," IEEE Trans. Antennas Propag., vol. 62, no. 5, pp. 2501-2513, May 2016.

[6] M.D. Migliore, "Minimum Trace Norm Regularization (MTNR) in electromagnetic inverse problems," IEEE Trans. Antennas Propag., vol. 64, no. 2, pp.630-639, Feb. 2016.

[7] J. Duchon, "Splines minimizing rotation-invariant semi-norms in Sobolev spaces," in W. Schempp and K. Zeller, editors, Constructive Theory of Functions of Several Variables, Springer-Verlag, 1987.

[8] J. Meinguet, "Multivariate Interpolation at Arbitrary Points Made Simple," Journal of Applied Mathematics and Physics, vol. 30, 1979

[9] E.J. Candès and B. Recht, "Exact Matrix Completion via Convex Optimization," Foundations of Computational Mathematics, vol. 9, no. 6 pp.712-772, Dec. 2009
[10] M. Fazel, "Matrix rank minimization with applications," Ph.D. dissertation, Stanford University, 2002.

[11] M. Fazel, H. Hindi, and S. Boyd, "Rank minimization and applications in system theory," American Control Conf., Boston, MA, USA, Jun. 2004

[12] B. Recht, M. Fazel, and P.A. Parrilo "Guaranteed Minimum Rank Solutions to Linear Matrix Equations via Nuclear Norm Minimization," SIAM Review, vol. 3, no. 52, pp.471-501, 2010.

[13] CVX Research, Inc. CVX: Matlab software for disciplined convex programming, version 2.0 beta. http://cvxr.com/cvx, September 2012.

[14] O.M. Bucci and G. Franceschetti, "On the degrees of freedom of scattered fields," IEEE Trans. Antennas Propag., vol. 37, no. 7, pp.918926, July 1989.

[15] M.D. Migliore, "On the Role of the Number of Degrees of Freedom of the Field in MIMO Channels," IEEE Trans. Antennas Propag., Vol. 54 no. 2, pp. 620-628, Feb. 2006.

[16] M.D. Migliore, "On electromagnetics and information theory," IEEE Trans. Antennas Propag., vol. 56, no. 10, pp. 3188-3200, Oct. 2008.

[17] O.M. Bucci, C. Gennarelli, and C. Savarese, "Fast and accurate nearfield far-field transformation by sampling interpolation of plane-polar measurements," IEEE Trans. Antennas Propag., vol. 39, pp.48-55, Jan. 1991.

[18] F.L. Bookstein, "Principal Warps: Thin-Plate Splines and the Decomposition of Deformations," IEEE Trans. Pattern Anal. Mach. Intell., vol. 11 no. 6, 567-585, June 1989. 